\title{
NuSTAR observations of heavily obscured Swift/BAT AGNs: Constraints on the Compton-thick AGNs fraction
}

\author{
I. Georgantopoulos and A. Akylas
}

\author{
IAASARS, National Observatory of Athens, I. Metaxa \& V. Pavlou, 15236, Greece \\ e-mail: ig@noa.gr
}

Received 16 March 2018 / Accepted 31 August 2018

\begin{abstract}
The evolution of the accretion history of the Universe has been studied in unprecedented detail owing to recent X-ray surveys performed by Chandra and XMM-Newton. A focus on the most heavily obscured or Compton-thick active galactic nuclei (AGNs) is missing in these studies. These AGNs evade detection even in X-ray surveys owing to their extreme hydrogen column densities, which exceed $10^{24} \mathrm{~cm}^{-2}$. Recently, the all-sky hard X-ray survey performed by Swift/BAT brought a breakthrough, allowing the detection of many of these AGNs. This is because of the very high energy bandpass (14-195 keV) of this instrument, which helps to minimise attenuation effects. In our previous work, we identified more than 50 candidate Compton-thick AGNs in the local Universe, corresponding to an observed fraction of about $7 \%$ of the total AGNs population. This number can only be converted to the intrinsic Compton-thick AGNs number density if we know their exact selection function. This function sensitively depends on the form of the Compton-thick AGN spectrum, that is the energy of their absorption turnover, photon-index and its cut-off energy at high energies, and the strength of the reflection component on the matter surrounding the nucleus. For example, the reflection component at hard energies 20-40 keV antagonises the number density of missing Compton-thick AGNs in the sense that the stronger the reflection the easier these sources are detected in the BAT band. In order to constrain their number density, we analysed the spectra of 19 Comptonthick AGNs that have been detected with Swift/BAT and have been subsequently observed with NuSTAR in the 3-80 keV band. We analysed their X-ray spectra using the MYTORUS models which properly take into account the Compton scattering effects. These were combined with physically motivated Comptonisation models, which accurately describe the primary coronal X-ray emission. We derived absorbing column densities that are consistent with those derived by the previous Swift/BAT analyses. We estimate the coronal temperatures to be roughly between 25 and $80 \mathrm{keV}$ corresponding to high energy cut-offs roughly between 75 and $250 \mathrm{keV}$. Furthermore, we find that the majority of our AGNs lack a strong reflection component in the $20-40 \mathrm{keV}$ band placing tighter constraints on the intrinsic fraction of Compton-thick AGNs. Combining these results with our X-ray background synthesis models, we estimate a percentage of Compton-thick AGNs in the local Universe of $\approx 20 \pm 3 \%$ relative to the type-II AGNs population.
\end{abstract}

Key words. X-rays: general - X-rays: diffuse background - X-rays: galaxies - quasars: supermassive black holes

\section{Introduction}

The vast majority of galaxies contain a supermassive black hole in their centres (e.g. Ferrarese \& Merritt 2000). A fraction of these emit copious amounts of radiation as in-falling matter forms an accretion disc around the black hole. These active galactic nuclei (AGNs) constitute at least half of the galaxy population (Ho et al. 1997a,b). The majority of AGNs are covered by veils of dust and gas (e.g. Ricci et al. 2017), which probably have a toroidal form (Wada et al. 2009). In most cases this obscuring screen can obliterate the optical emission and reradiate in the IR. X-rays can more easily penetrate these obscuring screens. However, in the most extreme obscuration even the X-ray radiation can be blocked. These Compton-thick AGNs have column densities that exceed $10^{24} \mathrm{~cm}^{-2}$. In this case, the X-rays are Compton scattered on the free electrons of the obscuring screen. As these heavily obscured AGNs represent the evasive side of the accretion history of the Universe, it is of primary importance to detect these objects. However, the methods employed to constrain their number appear to give diverging results.

The most commonly used method is that of X-ray background synthesis models. The spectrum of the X-ray background, that is the integrated X-ray light produced by all X-ray sources in the history of the Universe, presents a peak at $20-30 \mathrm{keV}$ as measured by the HEAO-1, XMMNewton, BeppoSAX, and the INTEGRAL missions (e.g. Gruber et al. 1999; Frontera et al. 2007; Churazov et al. 2007; Moretti et al. 2009). X-ray background synthesis models attempt to reconstruct the spectrum of the X-ray background using the AGN luminosity function, together with a model for the AGN X-ray spectrum. These models have been pioneered by Comastri et al. (1995) and were later refined by Gilli et al. (2007), Treister et al. (2009), Draper \& Ballantyne (2009), Akylas et al. (2012), Vasudevan et al. (2013), Ueda et al. (2014), and Esposito \& Walter (2016). A significant number of Compton-thick AGNs are necessary to reproduce the hump of the X-ray background. However, their fraction remains uncertain with figures ranging roughly between $10 \%$ and $50 \%$. This discrepancy is attributed mainly to the fact that there is a degeneracy between the required fraction of Compton-thick AGNs and the strength of the total AGNs population reflection component. This reflection component originates from scattering and subsequent absorption of the X-ray radiation on surrounding cold material (e.g. George \& Fabian 1991) and results in the flattening of the observed AGN spectrum. The higher the AGNs 
population reflection component the lower the number of Compton-thick AGNs are required. Interestingly enough, the reflection component strength does not refer to the Comptonthick AGNs but to the average reflection component of the total AGNs population.

Recently, attention has shifted to the direct detection of Compton-thick AGNs using X-ray spectroscopy. The sensitive observations performed with Chandra and XMM-Newton allowed the detection of many faint candidate Compton-thick AGNs in the 2-10 keV band, mainly in the Chandra Deep Field South area (e.g. Georgantopoulos et al. 2013; Brightman et al. 2014; Buchner et al. 2015), resulting in the derivation of the Compton-thick AGN luminosity function and its evolution. Additional studies have focussed on the ultra-hard band above $10 \mathrm{keV}$ where the X-ray attenuation is less severe. The Neil Gehrels Swift, INTEGRAL, and NuSTAR missions, which operate in this band, readily provide this possibility. The burst alert telescope (BAT) hard X-ray instrument on board Neil Gehrels Swift is continuously performing an all-sky survey in the $14-195 \mathrm{keV}$ band. As this instrument does not carry imaging X-ray optics it can probe flux limits of only a few times $10^{-12} \mathrm{erg} \mathrm{cm}^{-2} \mathrm{~s}^{-1}$. Ricci et al. (2015) and Akylas et al. (2016) analysed the X-ray spectra of 688 candidate AGNs in the 70month BAT all-sky survey (Baumgartner et al. 2013). Fitting the X-ray spectra using Bayesian statistics, Akylas et al. (2016) found about 50 Compton-thick AGNs candidates in overall agreement with the results of Ricci et al. (2015). The observed fraction of Compton-thick AGNs amounts to about $7 \%$ of the total AGNs population. This number can be extrapolated to the intrinsic fraction of Compton-thick AGNs, only by knowing the exact X-ray spectrum of Compton-thick AGNs, i.e. their selection function. Akylas et al. (2016) found that the intrinsic fraction of Compton-thick AGNs in the local Universe is between $10 \%$ and $30 \%$, assuming a reflection component that contributes between 0 and $5 \%$ to the total $2-10 \mathrm{keV}$ flux. These estimates are in good agreement with the estimates of Ricci et al. (2015), Burlon et al. (2011), and Malizia et al. (2009). The advantage of the above direct methods is that only knowledge of the $\mathrm{X}$-ray spectrum of Compton-thick AGNs is required instead of the average AGNs population.

In this paper, we present an X-ray spectral analysis of the Compton-thick AGNs in Akylas et al. (2016), which has publicly available NUSTAR spectra. The imaging X-ray optics of NuSTAR (Harrison et al. 2013) allow the derivation of excellent quality spectra. These are used to constrain with the highest accuracy yet, the fraction of Compton-thick AGNs in the local Universe. We adopt $\mathrm{H}_{\mathrm{o}}=75 \mathrm{~km} \mathrm{~s}^{-1} \mathrm{Mpc}^{-1}, \Omega_{M}=0.3$, and $\Omega_{\Lambda}=0.7$ throughout the paper.

\section{X-ray sample and data}

\subsection{Burst Alert Telescope}

The Neil Gehrels Swift gamma-ray burst (GRB) observatory (Gehrels et al. 2004) was launched in November 2004 and has been continually observing the hard X-ray (14-195 keV) sky with the BAT instrument. The BAT is a large, coded-mask telescope optimised to detect transient GRBs and is designed with a very wide field of view of $\sim 60 \times 100^{\circ}$. The observation strategy of Neil Gehrel Swift is to observe targets with the narrow fieldof-view X-ray telescope (XRT), in the $0.3-10 \mathrm{keV}$ band until a new GRB is discovered by BAT, at which time Neil Gehrel Swift automatically slews to the new GRB to follow up with the narrow field instruments until the $\mathrm{X}$-ray afterglow is below the XRT detection limit. Baumgartner et al. (2013) presented the catalogue of sources detected in 70 months of observations with BAT. The BAT 70 month survey has detected 1171 hard X-ray sources down to a flux level of $10^{-11} \mathrm{erg} \mathrm{cm}^{-2} \mathrm{~s}^{-1}$. The majority of these sources are AGNs.

Akylas et al. (2016) have analysed the X-ray spectra of these sources employing Bayesian statistics. These authors considered 688 sources classified according to the NASA/IPAC Extragalactic Database in the following types: (i) 111 galaxies, (ii) 292 Seyfert I (Sy 1.0-1.5), (iii) 262 Seyfert II (Sy 1.7-2.0), and (iv) 23 sources of other types of AGNs. Radio-loud AGNs were excluded since their X-ray emission might be dominated by the jet component. Quasi Stellar Objects (QSOs) have been also excluded from the analysis since the fraction of highly absorbed sources within this population is negligible. These authors have combined the BAT with the XRT data $(0.3-10 \mathrm{keV})$ at softer energies adopting a Bayesian approach to fit the data using Markov chains. This allowed us to consider all sources as potential Compton-thick candidates at a certain level of probability. Fifty-three sources present a non-zero probability of being Compton-thick corresponding to 40 effective Comptonthick sources. These represent $7 \%$ of the sample in excellent agreement with the figure reported in Ricci et al. (2015) and Burlon et al. (2011). Out of these, 38 sources have a probability of more than $70 \%$ being Compton-thick.

\subsection{NUSTAR}

The Nuclear Spectroscopic Telescope Array, NuSTAR, (Harrison et al. 2013) launched in June 2012, is the first orbiting X-ray observatory that focusses light at high energies $(E>10 \mathrm{keV})$. It consists of two co-aligned focal plane modules (FPMs), which are identical in design. Each FPM covers the same $12 \times 12$ arcmin portion of the sky and comprises four cadmium-zinc-telluride detectors. The NuSTAR array operates between 3 and $79 \mathrm{keV}$ and provides an improvement of at least two orders of magnitude in sensitivity compared to previous hard X-ray orbiting observatories, $E>10 \mathrm{keV}$. This is because of its excellent spatial resolution, which is 58 arcsec half-power diameter. The energy resolution is 0.4 and $0.9 \mathrm{keV}$ at 6 and $60 \mathrm{keV}$, respectively.

All the 38 candidate Compton-thick objects in Akylas et al. (2016) with a probability $>70 \%$ have either been observed or are scheduled for observation with $N U S T A R$. We analysed the 19 sources that are currently publicly available. The details of the NUSTAR observations for each object are given in Table 1. We extracted the spectra from each FPM using a circular aperture region of 30 arcsec radius corresponding to $\sim 50 \% \mathrm{NuSTAR}$ encircled energy fraction (ECF). We processed the data with the NuSTAR Data Analysis Software (NUSTARDAS) v1.4.1within HEASOFT v6.15. The NUPIPELINE script was used to produce the calibrated and cleaned event files using standard filter flags. We extracted the spectra and response files using the NUPRODUCTS task.

\section{X-ray spectral modelling}

Recently, sophisticated spectral models have been developed that estimate the detailed spectra of Compton-thick AGNs, that is they take into account Compton scattering and reprocessing by Compton-thick material that surrounds the nucleus. These include the models of Brightman \& Nandra (2011) and the models of Yaqoob \& Murphy (2011). Both models employ Monte Carlo simulations to account for Compton scattering 
Table 1. NUSTAR observations.

\begin{tabular}{lccccc}
\hline \hline Name & Redshift & $\begin{array}{c}\text { Gal. } N_{\mathrm{H}} \\
\times 10^{20} \mathrm{~cm}^{-2}\end{array}$ & obsID & $\begin{array}{c}\text { Exposure } \\
\text { ksec }\end{array}$ & $\begin{array}{c}\text { Net counts } \\
3-79 \mathrm{keV}\end{array}$ \\
\hline NGC 1068 & 0.0038 & 2.9 & 60002030002 & 57.8 & 18900 \\
Circinus & 0.0014 & 5.6 & 60002039002 & 53.9 & 87962 \\
NGC 6240 & 0.0244 & 4.9 & 60002040002 & 30.9 & 8716 \\
NGC 4945 & 0.0019 & 13.9 & 60002051004 & 54.6 & 45725 \\
NGC 424 & 0.0118 & 1.6 & 60061007002 & 15.5 & 1592 \\
2MFGC2280 & 0.0151 & 40.6 & 60061030002 & 15.9 & 640 \\
NGC 1194 & 0.0136 & 7.1 & 60061035002 & 31.5 & 4060 \\
MGC06-16-028 & 0.0157 & 6.2 & 60061072002 & 23.6 & 1649 \\
NGC 3079 & 0.0037 & 0.8 & 60061097002 & 21.5 & 1470 \\
NGC 3393 & 0.0125 & 6.0 & 60061205002 & 15.7 & 1609 \\
NGC 4941 & 0.0037 & 2.4 & 60061236002 & 20.7 & 1491 \\
NGC 5728 & 0.0037 & 7.8 & 60061256002 & 24.4 & 7344 \\
ESO137-34 & 0.0091 & 24.7 & 60061272002 & 18.5 & 2043 \\
NGC 7212 & 0.0266 & 5.5 & 60061310002 & 24.6 & 1464 \\
NGC 1229 & 0.03623 & 1.7 & 60061325002 & 24.9 & 1700 \\
NGC 6232 & 0.0148 & 4.5 & 60061328002 & 18.1 & 284 \\
2MASSXJ09235371-3141305 & 0.0424 & 12.8 & 60061339002 & 21.3 & 2940 \\
NGC 5643 & 0.0040 & 8.3 & 60061362002 & 22.5 & 1664 \\
NGC 7130 & 0.0161 & 1.9 & 60261006002 & 42.1 & 1302 \\
\hline
\end{tabular}

and the geometry of the obscuring screen. The models of Brightman \& Nandra (2011) refer to both spherical coverage and a torus geometry. These estimate self-consistently the Compton scattering along the line of sight as well as the reflection from surrounding matter. Instead, the toroidal Compton-thick X-ray re-processor model, MYTORUS of Murphy \& Yaqoob (2009) and Yaqoob \& Murphy (2011), uses a torus geometry. The torus has a diameter that is characterised by the equatorial column density, $N_{\mathrm{H}}, \mathrm{Z}$. The model assumes a configuration in which the global covering factor of the re-processor is 0.5 , corresponding to a solid angle subtended by the torus of $2 \pi$. The Yaqoob \& Murphy (2011) model employs three separate components: the line of sight component, $Z$, an absorbed reflected component along the line of sight, $S 90$, and an unabsorbed reflected component S0. In our spectral fits, we chose to employ the spectral models of Yaqoob \& Murphy (2011). This is because this model allows for free relative normalisations between the different components in contrast to the self-consistent models of Brightman \& Nandra (2011). Then the Yaqoob \& Murphy (2011) model can accommodate differences in the actual geometry and time delays between direct, scattered, and fluorescent line photons. We note that the reflected emission is modelled using the appropriate column density instead of being modelled using reflection on a slab of infinite column density, cf. the PEXRAV model Magdziarz \& Zdziarski (1995). The full general geometrical set-up of this model can be visualised in Fig. 2 of Yaqoob (2012).

In detail, our modelling consists of the following components. a) An unabsorbed power law originates from scattered emission on Compton-thin material, usually referred as the "soft excess" in type-2 AGNs b) The direct zeroth order primary emission absorbed by the line-of-sight column $N_{\mathrm{H}}, Z$ is denoted by the multiplicative model mytorus_Ezero. Following Yaqoob (2012), we used the physically motivated model COMPTT of Titarchuk (1994). This model estimates the scattering of soft accretion disc photons on a hot thermal corona with temperature kT and optical depth $\tau$. According to this thermal Comptonisation model the resulting cut-off energy is $\sim 3 \mathrm{kT}$. There is dependence between the optical depth and temperature of the corona in the sense that the higher the temperature of the corona the lower the value of $\tau$. In the case where the optical depth cannot be constrained from the spectral fits, we chose to fix the value of the optical depth to $\tau=2$. This value corresponds roughly to a photon index of $\Gamma \approx 1.9$ for a coronal temperature of $k T \sim 50 \mathrm{keV}$ (the average value estimated in our spectral fits below) in the case of spherical geometry (Titarchuk 1994; Longair 2011). The mean photon-index value observed in AGNs is $\Gamma=1.9$ (Nandra \& Pounds 1994) and is also the best fit photon index found in our X-ray background synthesis models Akylas et al. (2012). c) A reflected absorbed component S90 with a column density $N_{\mathrm{H}}, \mathrm{S} 90$ is denoted by the model mytorus_scattered. d) A reflected unabsorbed component, S0, from a column density $N_{\mathrm{H}}$, S0 could possibly originate from the back-side of the torus. e) An FeK $\alpha, \mathrm{FeK} \beta$, and ionised Fe lines at $6.4,7.02$ and $6.7 \mathrm{keV}$, respectively, is denoted by the model MYTL arising from the line of sight absorbed scattered radiation. $f$ ) The same lines as above are denoted again by the model MYTL arising from unabsorbed scattered radiation. We assume that all the above column densities have the same value. The normalisations of the two scattered components and of the zeroth order component are allowed to vary independently. In XSPEC notation, the model can be written as

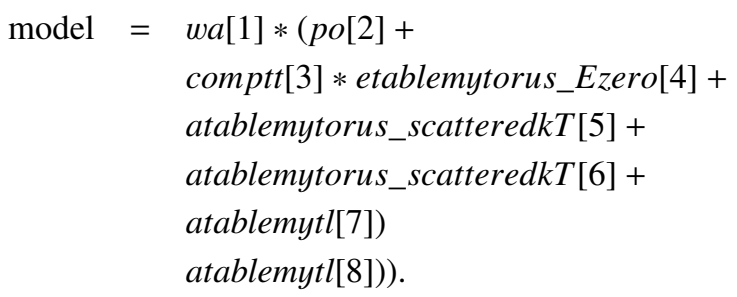

The scattering models mytorus_scatteredkT and atablemytl correspond to a temperature $k T$ of the corona obtaining discrete values between 16 and $100 \mathrm{keV}$. The use of these models requires an iterative process. More specifically, it is necessary to have a priori knowledge of the primary radiation spectrum and 
Table 2. MYTORUS Spectral fits.

\begin{tabular}{lccccccc}
\hline \hline $\begin{array}{l}\text { Name } \\
(1)\end{array}$ & $\begin{array}{c}N_{\mathrm{H}}, Z \\
(2)\end{array}$ & $\begin{array}{c}k T \\
(3)\end{array}$ & $\begin{array}{c}\tau \\
(4)\end{array}$ & $\begin{array}{c}E_{\mathrm{C}} \\
(5)\end{array}$ & $\begin{array}{c}f_{2}^{\text {refl }} 0-40 \mathrm{keV} \\
(6)\end{array}$ & $\begin{array}{c}\chi^{2} / \text { d.o.f. } \\
(7)\end{array}$ & $\begin{array}{c}\text { Ref. } \\
(8)\end{array}$ \\
\hline NGC 1068 & $>10$ & $67_{-28.0}^{+97.0}$ & $0.68_{-0.23}^{+0.08}$ & $>145$ & 0.84 & $890 / 755$ & $\mathrm{a}, \mathrm{i}$ \\
Circinus & $9.0_{-0.50}^{+1.00}$ & $38_{-7.5}^{+9.5}$ & $0.61_{-0.08}^{+0.10}$ & $43_{-4}^{+5}$ & 0.80 & $1872 / 1724$ & $\mathrm{~b}$ \\
NGC 6240 & $1.3_{-0.22}^{+0.12}$ & $60_{-20.5}^{+85.5}$ & $1.7_{-0.73}^{+0.23}$ & unconstr. & $<0.01$ & $456 / 496$ & $\mathrm{c}, \mathrm{i}$ \\
NGC 4945 & $3.5_{-0.13}^{+0.20}$ & $59_{-13.3}^{+19.9}$ & $1.7_{-0.32}^{+0.34}$ & $>42$ & 0.02 & $1786 / 1743$ & $\mathrm{~d}, \mathrm{i}$ \\
NGC 424 & $1.3_{-0.20}^{+0.25}$ & $30_{-12.1}^{+20.9}$ & 2 & $>64$ & 0.93 & $147 / 121$ & $\mathrm{e}, \mathrm{i}$ \\
2MFGC2280 & $1.6_{-0.45}^{+0.72}$ & 50.13 & $1.3_{-1.3}^{+1.4}$ & $<238$ & 0.03 & $66 / 59$ & $\mathrm{i}$ \\
NGC 1194 & $1.7_{-0.25}^{+0.24}$ & $37_{-1.0}^{+4.7}$ & $1.5_{-0.13}^{+0.30}$ & unconstr. & $<0.01$ & $257 / 273$ & $\mathrm{f}$ \\
MCG+06-16-028 & $1.5_{0.27}^{+0.42}$ & $34_{-18.1}^{+20.0}$ & $1.4_{-1.3}^{+0.7}$ & $>23$ & 0.04 & $136 / 132$ & $\mathrm{i}$ \\
NGC 3079 & $3.2_{-0.43}^{+0.54}$ & $31_{-8.1}^{+8.6}$ & 2 & $56_{-24}^{+354}$ & 0.05 & $192 / 165$ & $\mathrm{i}$ \\
NGC 393 & $2.1_{-0.28}^{+0.61}$ & $69_{-60.9}^{+77.9}$ & $1.05_{-1.05}^{+0.97}$ & $>96$ & $<0.01$ & $123 / 117$ & $\mathrm{~g}, \mathrm{i}$ \\
NGC 4941 & $1.2_{-0.39}^{+0.91}$ & $47_{-34}^{+216}$ & 2 & unconstr. & 0.19 & $98 / 111$ & \\
NGC 5728 & $1.3_{-0.08}^{+0.13}$ & $81_{-30.2}^{+48.6}$ & $1.5_{-0.41}^{+0.32}$ & $121_{-13}^{+11}$ & $<0.01$ & $444 / 436$ & $\mathrm{i}$ \\
ESO137-34 & $2.9_{-0.54}^{+0.86}$ & $26_{-11.8}^{+15.9}$ & 2 & $>203$ & 0.12 & $175 / 147$ & $\mathrm{i}$ \\
NGC 7212 & $1.1_{-0.27}^{+0.48}$ & $41_{-22.6}^{+32.2}$ & 2 & $>56$ & $<0.01$ & $147 / 117$ & $\mathrm{i}$ \\
NGC 1229 & $0.6_{-0.14}^{+0.26}$ & $60_{-22.5}^{+51.2}$ & 2 & unconstr. & $<0.01$ & $134 / 138$ & $\mathrm{i}$ \\
NGC 6232 & $0.8_{-0.30}^{+1.26}$ & $42_{-29.5}^{+40.2}$ & 2 & $>500$ & $<0.01$ & $52 / 35$ & $\mathrm{i}$ \\
2MASXJ09235371 & $1.0_{-0.18}^{+0.21}$ & $60_{-27.9}^{+68.1}$ & 2 & $35_{-5.1}^{+6.1}$ & $<0.01$ & $266 / 209$ & $\mathrm{i}$ \\
NGC 5643 & $1.3_{-0.10}^{+0.10}$ & 50 & 2 & $>48$ & 0.92 & $136 / 127$ & $\mathrm{~h}, \mathrm{i}$ \\
NGC 7130 & $2.3_{-0.31}^{+0.29}$ & 50 & 2 & unconstr. & 0.23 & $116 / 126$ & $\mathrm{i}$ \\
\hline
\end{tabular}

Notes. (1) Source name; (2) average column density $\left(N_{\mathrm{H}}, Z=N_{\mathrm{H}}, \mathrm{S} 90=N_{\mathrm{H}}, \mathrm{S} 0\right)$ in units of $10^{24} \mathrm{~cm}^{-2}$; (3) coronal temperature (keV); values with no error bar were unconstrained and have been fixed to a value of $50 \mathrm{keV}$. (4) optical depth; values with no error bars were unconstrained and have been fixed to a value of $\tau=2$. (5) the high energy cut-off derived by Ricci et al. (2017) from Neil Gehrels Swift, $X M M-N e w t o n, C h a n d r a$ joint fits; and (6) unobscured reflected fraction in the $20-40 \mathrm{keV}$ band. The unobscured reflection fraction is defined as Flux $[\mathrm{S} 0] /\left(\right.$ Flux$[\mathrm{Z}]+$ Flux[S90] + Flux[S0]); (8) $\chi^{2}$ over degrees of freedom. (9) References for previous NuSTAR analyses.

References. (a) Bauer et al. (2015); (b) Arévalo et al. (2014) ; (c) Puccetti et al. (2014); (d) Puccetti et al. (2016); (e) Baloković et al. (2014); (f) Masini et al. (2016) (g) Koss et al. (2015) (h) Annuar et al. (2015) (i) Marchesi et al. (2018).

in particular of the cut-off to estimate the output reflection spectrum. In the MYTORUS models, this is a free parameter in the zeroth component spectrum but not in the reflection spectra. In the first run we give an initial cut-off energy of $50 \mathrm{keV}$ as an input to estimate the reflection components S0 and S90. Then the exact cut-off energy is estimated from the fit to the zeroth order component. This value is fed back to the reflection components and the process is repeated. In the above process we leave the NUSTAR and BAT normalisations untied. We use the XSPEC v12.8 spectral fitting package (Arnaud et al. 1996). The appropriate Galactic absorption column density has been included in all fits. Owing to the high number of counts we employ the $\chi^{2}$ statistic. We bin the spectra, using the task GRPPHA so that they contain at least 20 counts per bin. All errors quoted refer to the $90 \%$ confidence level.

\section{Results}

The spectral fitting results are given in Table 2. In the following subsections we discuss separately our findings regarding the hydrogen column density, the high energy cut-off and the reflection fraction. Finally, we discuss the implications of our spectral modelling for the intrinsic number of Compton-thick AGNs

\subsection{Absorbing column density}

The distribution of the column densities is presented in Fig. 1. The column densities found by NUSTAR are in very good agreement with those previously found by Neil Gehrels Swift

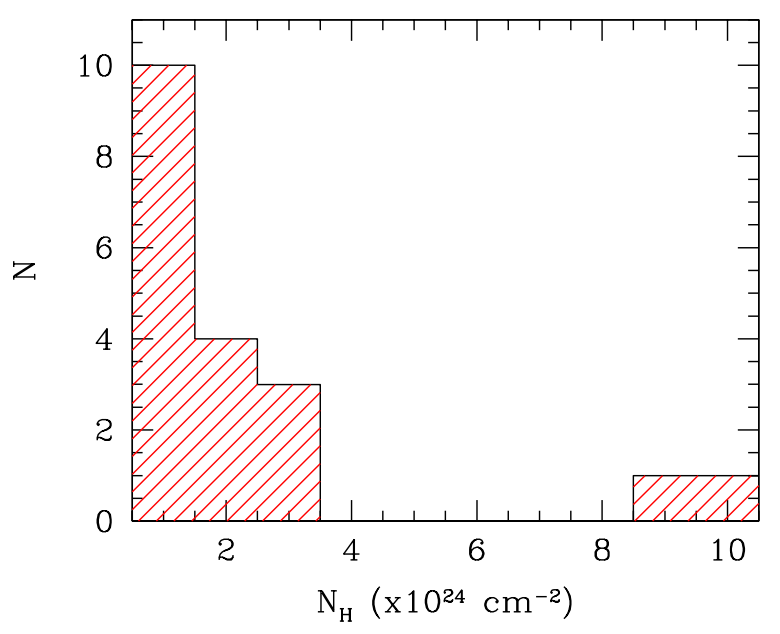

Fig. 1. Column density distribution for all 19 sources in our sample.

(Akylas et al. 2016). Akylas et al. (2016) found a much higher column density for only one source (NGC 7212). The current NUSTAR results confirm the lower column density reported by Ricci et al. (2015). This difference is most probably because the Neil Gehrels Swift/XRT data have limited photon statistics while Ricci et al. (2015) have combined the BAT data with XMM-Newton spectra obtaining better quality photon statistics at energies below $10 \mathrm{keV}$. Recently, Marchesi et al. (2018) published a NUSTAR analysis of Compton-thick AGNs 
from the Neil Gehrels Swift/BAT list of Ricci et al. (2015). Marchesi et al. (2018) found that a number of candidate Compton-thick AGNs in Ricci et al. (2015) may present lower column densities. There are 15 common objects between our sample and that of Marchesi et al. (2018). According to these authors four objects appear to have column densities somewhat lower than $10^{24} \mathrm{~cm}^{-2}$. These are NGC 1194, NGC 6232, NGC 1229, and 2MASSXJ09235371-3141305. According to our NUSTAR analysis, NGC 1194 is above the Compton-thick threshold while NGC 1229 and 2MASSXJ09235371-3141305 are consistent with being Compton-thick within the errors. In the case of NGC 1229, although the source is unambiguously heavily obscured $\left(N_{\mathrm{H}} \approx 6_{-1.4}^{+2.6} \times 10^{23} \mathrm{~cm}^{-2}\right)$ the column density derived lies well below the Comptonthick limit. For many other sources, for example NGC 1068, Circinus, NGC 424, NGC 3079, NGC 4945, and NGC 5643, NuSTAR spectral fits have been presented elsewhere (Bauer et al. 2015; Arévalo et al. 2014; Baloković et al. 2014; Puccetti et al. 2014, 2016; Koss et al. 2015; Annuar et al. 2015; Marchesi et al. 2018). In general, our results are consistent with the work cited above.

As can be seen from Fig. 1, there is a clear tendency to detect mildly obscured Compton-thick AGNs with column densities a few times $10^{24} \mathrm{~cm}^{-2}$. This has been first noticed by Burlon et al. (2011) who showed that the BAT instrument is unbiased only up to column densities of only $2 \times 10^{24} \mathrm{~cm}^{-2}$. We observe only two heavily Compton-thick source with a column density around $10^{25} \mathrm{~cm}^{-2}$. Owing to their diminished X-ray emission, these heavily obscured sources can be detected only if they are relatively nearby. A similar effect is observed in INTEGRAL observations of Compton-thick AGNs (Malizia et al. 2009). Although the number of Compton-thick AGNs is relatively limited in the current sample, Akylas et al. (2016) using a much larger sample noticed that the column density distribution is fully consistent with a flat Compton-thick column density distribution between $10^{24}$ and $10^{26} \mathrm{~cm}^{-2}$.

\subsection{High energy cut-off}

In column (3) of Table 2 we give the derived coronal temperature. The cut-off energy corresponds to $\sim 3 k T$ (Titarchuk 1994). For three objects the coronal temperature could not be constrained (2MFGC2280, NGC 5643, and NGC 7130). The mean energy of the coronal temperature of the remaining 16 sources is $k T \approx 50 \mathrm{keV}$ corresponding to a high energy cut-off of about $\mathrm{E}_{\mathrm{C}} \sim 150 \mathrm{keV}$. The distribution of the coronal temperature is given in Fig. 3. In the case of the three sources above where the coronal temperature could not be well constrained, we fixed the temperature at $50 \mathrm{keV}$ to estimate the reflected emission.

It is interesting to compare the estimated cut-off with other high energy spectral studies of Compton-thick AGNs. Dadina (2007) estimated the cut-off energy in a number of nearby AGNs using data from the BeppoSAX mission, which observes in the $2-100 \mathrm{keV}$ range. There are eight sources in common between our sample and that of Dadina (2007). Owing to the limited photon statistics at high energies, Dadina (2007) were able to constrain the high-energy cut-off in only two of these sources: NGC 1068, with $E_{\mathrm{C}}>116 \mathrm{keV}$, and NGC 4945, with $E_{\mathrm{C}}=122_{-26}^{+41} \mathrm{keV}$. These values are entirely consistent with our combined NuSTAR and BAT analysis. Ricci et al. (2017) derived the cut-off energy of the Compton-thick AGNs in his sample using a combination of XMMNewton, Chandra, and Neil Gehrels Swift spectra (both XRT and BAT). For comparison with the present work, the cut-off energies, $E_{\mathrm{C}} \sim 3 k T$, derived by Ricci et al. (2017), are given in column (5) of Table 2. In many cases, there is reasonable agreement between the two analyses. However, there are at least four cases in which the results are clearly at odds. These are Circinus, ESO137-34, NGC 6232, and 2MASXJ09235371. This discrepancy could be partly attributed to the different spectral models used, that is the power-law model with an exponential cut-off versus the Comptonisation models. Ricci et al. (2017) have determined the average cut-off energy for their Compton-thick sources. When they excluded the lower and upper limits, they found a very low high energy cut-off for their Compton-thick AGNs $\left(E_{\mathrm{C}} \approx 43 \pm 15 \mathrm{keV}\right)$. Taking lower and upper limits into account by means of the KaplanMeier estimator, they estimated a much higher value for the cutoff $E_{\mathrm{C}} \sim 450 \pm 64 \mathrm{keV}$. We found a mean high energy cut-off of $E_{\mathrm{C}} \sim 3 k T \approx 150 \mathrm{keV}$, excluding the three sources above where the cut-off energy could not be constrained. It is also interesting to compare with the work of Dadina (2007) who derived the average X-ray spectrum of 42 type-2 AGNs using BeppoSAX data. They found a cut-off energy of $E_{\mathrm{C}}=376 \pm 42 \mathrm{keV}$. This is significantly higher than the cut-off energy of our Compton-thick sources.

Our results are not significantly affected by the choice of $\tau=2.0$ in the cases where the photon statistics is limited. For example, in the case of NGC 3079 if we choose $\tau=1.5$, the resulting temperature becomes $k T=39 \pm 19 \mathrm{keV}$. For this temperature of the corresponding photon index would be $\Gamma \approx 2.0$.

\subsection{Reflection component}

The fraction of the reflected emission in the $20-40 \mathrm{keV}$ band plays an important role in the prediction of the intrinsic fraction of Compton-thick AGNs in the sense that as the reflected emission becomes higher, the number of Compton-thick AGNs required is reduced. This is because the higher the reflection the easier it is to detect Compton-thick AGNs in the BAT band and hence the number of missing Compton-thick AGNs is relatively small. The reflection fraction is given in column (6) of Table 2. This is defined as the ratio of the unabsorbed reflection component S0 versus the total flux, i.e. the sum of the line-of-sight component $Z$ and the two scattered components S0 and S90. Four sources appear to be reflection dominated as the reflection radiation fraction is near unity. These are NGC 1068, Circinus, NGC 424, and NGC 5643. A typical spectrum of a source with a strong reflection component (NGC 5643) is shown in Fig. 5. There is not a clear correlation between the strength of the reflection component and the column density in these sources. For example, NGC 424 and NGC 5643 have moderate Comptonthick column densities $1.3 \times 10^{24} \mathrm{~cm}^{-2}$. The vast majority of the sources have moderate strength reflection components below 0.23 . There are eight sources in which the best-fit reflection component is negligible i.e. well below 0.01 . The distribution of the reflection fraction is given in Fig. 2. The mean reflection fraction is 0.22 . In analogy to the commonly used PEXRAV model (Magdziarz \& Zdziarski 1995), which estimates the reflection to an infinite slab, we find that a fraction of 0.22 corresponds to a reflection strength $\mathrm{R} \approx 1$. This is equivalent to a reflection fraction of $\sim 3 \%$ in the $2-10 \mathrm{keV}$ band.

\subsection{Intrinsic fraction of Compton-thick AGNs}

In this section we estimate the intrinsic number of Comptonthick AGNs in the Universe using the population synthesis models developed by Akylas et al. (2012). The X-ray background synthesis models have been primarily devised to predict the number of Compton-thick AGNs based on their contribution to the X-ray background spectrum and in particular at its energy 


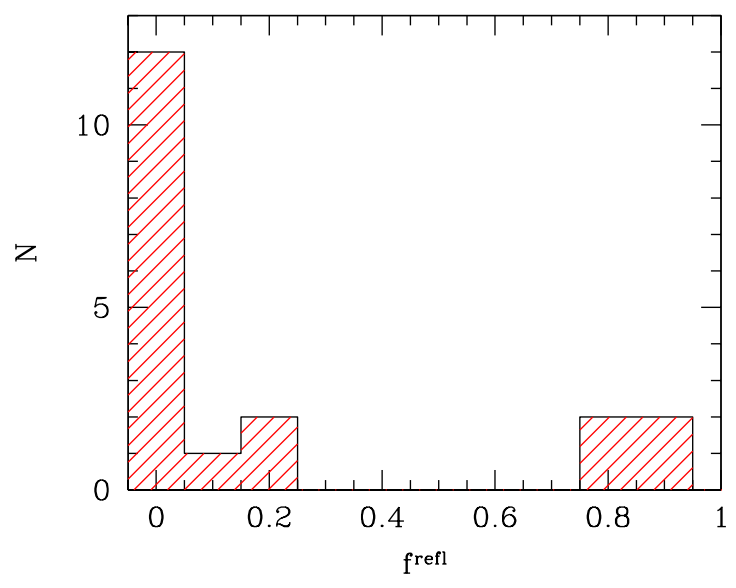

Fig. 2. Reflection fraction distribution $\left(f^{\mathrm{refl}}=\right.$ Flux $[\mathrm{S} 0] /(\mathrm{Flux}[\mathrm{Z}]+$ Flux[S90] + Flux[S0] ) in the $20-40 \mathrm{keV}$ band for all 19 sources in our sample.

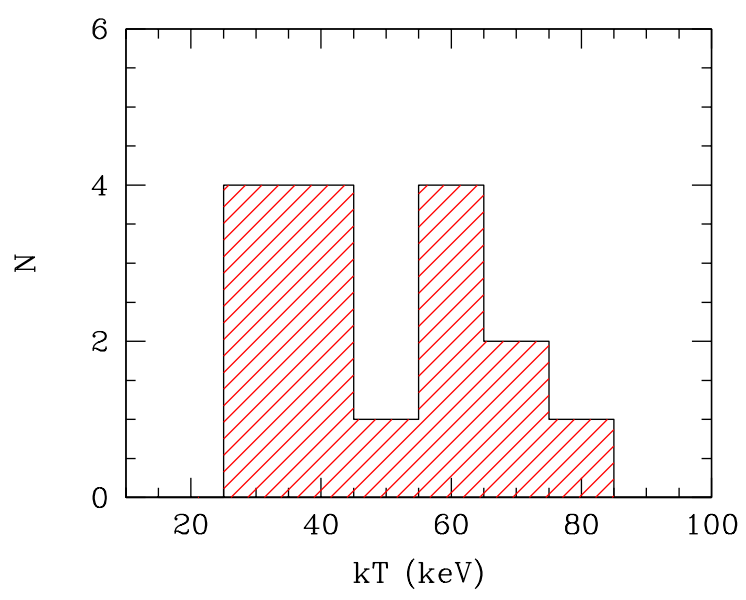

Fig. 3. Coronal temperature distribution as estimated using the COMPTT model of Titarchuk (1994; 16 sources). The three sources in which $k T$ is unconstrained have been excluded.

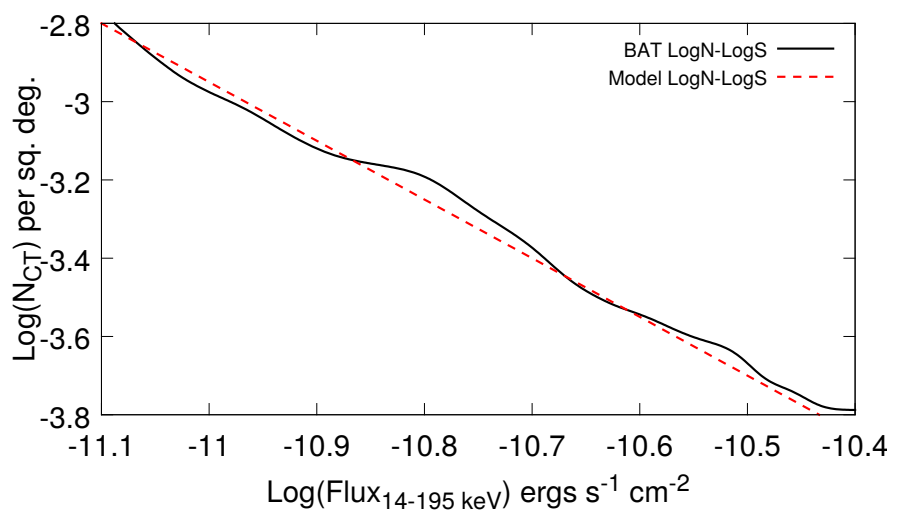

Fig. 4. Number count distribution of the Compton-thick AGNs derived from the Neil Gehrels Swift-BAT survey in Akylas et al. (2016; black solid line). The red dashed line denotes the prediction of the X-ray population synthesis model of Akylas et al. (2012). Using the spectral parameters found in our spectral fits, we find that the Compton-thick AGNs fraction should be $20 \%$ of the type- 2 AGNs population to agree with the observed $\log N-\log S$.
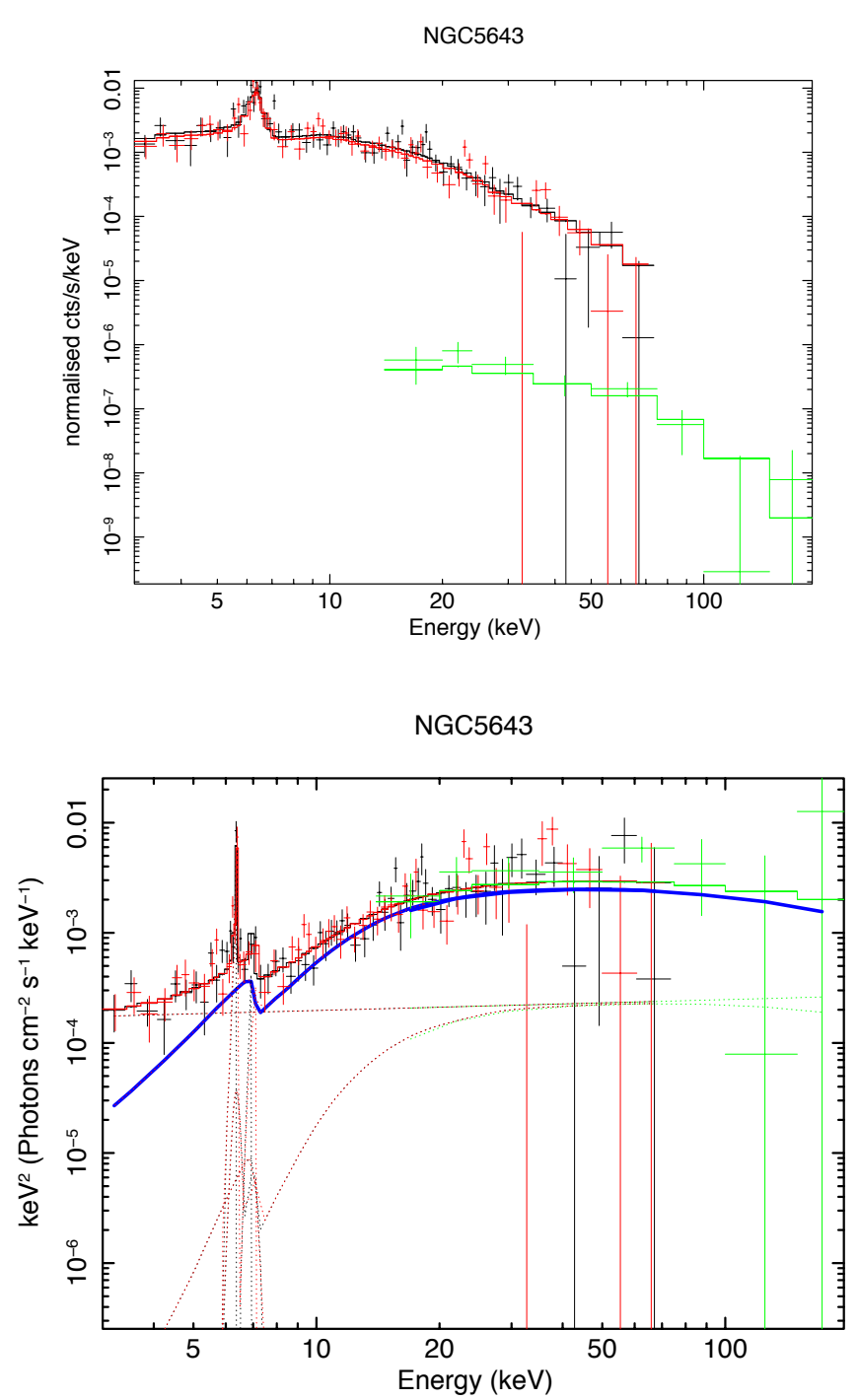

Fig. 5. NGC 5643: a characteristic spectrum of an AGN with a high reflection component. Top panel: folded spectrum. Bottom panel: unfolded spectrum; the blue sold line denotes the unabsorbed component S0. The straight dotted line represents the soft power-law component while the other dotted line denotes the absorbed reflection component S90. In both panels black and red denote the two FPM NuSTAR detectors while green corresponds to the BAT detector.

peak around $20-30 \mathrm{keV}$. The X-ray background synthesis models predict the number of AGNs and their contribution to the $\mathrm{X}$-ray background spectrum at any redshift, luminosity, and column density. The necessary ingredients are the AGN luminosity function and the typical AGN spectrum. Treister et al. (2009) first pointed out that uncertainties on the measurements of the $\mathrm{X}$-ray background are substantially large to hamper an accurate prediction of the number of Compton-thick AGNs. Instead these authors proposed that the INTEGRAL or BAT number counts of Compton-thick AGNs place tighter constraints. In this manner, the X-ray background synthesis models could be used to extrapolate the observed number counts to the intrinsic population number counts. In a similar approach, Burlon et al. (2011) used the observed Compton-thick AGNs number counts and predict the intrinsic Compton-thick AGNs counts by assuming a relation between the observed and intrinsic Comptonthick flux. This conversion sensitively depends on the typical 
Compton-thick AGN spectrum. Using a simple spectrum and by deriving the intrinsic $N_{\mathrm{H}}$ distribution, Burlon et al. (2011) derived an intrinsic Compton-thick AGN contribution of $\sim 20 \%$.

We fold the full observed spectral distribution as described by the parameters $N_{\mathrm{H}}, \mathrm{kT}$, and the reflection fraction (see Figs. 1-3) in our X-ray population synthesis models. Then, the remaining unknown parameter is the intrinsic fraction of Compton-thick AGNs. We find that in order to match the observed Compton-thick AGNs counts in the BAT 14-195 keV band with our models, we need an intrinsic fraction of $\sim 20 \%$ relative to the type-II AGNs population $\left(N_{\mathrm{H}}>10^{22} \mathrm{~cm}^{-2}\right)$; see Fig. 4. This corresponds to a Compton-thick fraction of $15 \%$ relative to the total AGNs population. The statistical error on this figure is $3 \%$. This is derived from the number of Compton-thick AGNs $(53 \pm 7)$ used to derive the $\log N-\log S$. The uncertainties on the spectral parameters estimated above have not been taken into account in this error estimate.

Recently, Masini et al. (2018) estimated the Compton-think fraction of Compton-thick sources, using NuSTAR observations in the UKIDSS Ultra deep field. These authors found an observed Compton-thick fraction of $8.4 \pm 2 \%$ in the $8-24 \mathrm{keV}$ band at a flux of $2.7 \times 10^{-14} \mathrm{erg} \mathrm{cm}^{-2} \mathrm{~s}^{-1}$. Making corrections for their non-detected $X M M-N e w t o n$ sources this fraction becomes $11.5 \pm 2.0$. This is in agreement with the $1 \sigma$ upper limit of our model predictions, which is $\approx 10 \%$ at this flux.

\section{Summary and conclusions}

Direct spectroscopic observations of AGNs in the local Universe, using Neil Gehrels Swift/BAT samples, find a fraction of Compton-thick AGNs of about $7 \%$ of the total AGN population (Burlon et al. 2011; Ricci et al. 2015; Akylas et al. 2016; Marchesi et al. 2018). This figure can be extrapolated to the intrinsic number of Compton-thick AGNs by assuming a spectral model for the Compton-thick AGN spectrum (e.g. Burlon et al. 2011). The spectral parameters of interest are the photon index, strength of the reflection component, column density distribution, and primary emission cut-off energy. The reflection of material on cold matter surrounding the nucleus results in the flattening of the spectrum around $20-30 \mathrm{keV}$. Therefore the higher the reflection the easier it is to detect Compton-thick AGNs in the $14-195 \mathrm{keV}$ band and hence the lower the number of missing Compton-thick AGNs (the lower the intrinsic fraction). The high energy cut-off works in the opposite way. The lower the value of the cut-off energy, the higher the intrinsic number of Compton-thick AGNs as these would not be easily detected in the BAT band. For example, if the high energy cut-off were $\approx 50 \mathrm{keV}$ (see e.g. Ricci et al. 2017), the fraction of Compton-thick AGNs would increase to about 50\%. We note that in contrast cut-offs higher than $150 \mathrm{keV}$ would not significantly affect our results.

Aiming to constrain the intrinsic fraction of Compton-thick AGNs in the local Universe, we explored the spectrum of a large number Compton-thick AGNs. We analysed the combined $\mathrm{NuS}$ $T A R /$ BAT spectra of 19 candidate Compton-thick AGNs (at the $70 \%$ confidence level) in the sample of Akylas et al. (2016). We modelled the spectra using the MYTORUS models of Yaqoob (2012), which take into account absorption and reflection from Compton-thick material. The primary X-ray emission from a hot corona is modelled with a physically motivated model (Titarchuk 1994). Our results can be briefly summarised as follows:

We derive absorbing column densities that are consistent with those derived by the previous Neil Gehrels Swift/BAT analyses by Ricci et al. (2015) and Akylas et al. (2016). We estimate that the coronal temperatures lie between 25 and $80 \mathrm{keV}$ corresponding to high energy cut-offs roughly between 75 and $240 \mathrm{keV}$. The mean cut-off energy is $150 \mathrm{keV}$. We find that a large fraction of our AGNs lack a significant reflection component in the $20-40 \mathrm{keV}$ band. The average reflection fraction is $\sim 0.22$. The resulting spectral parameters are used to place constraints on the intrinsic fraction of Compton-thick AGNs. Using the above spectral results in combination with our synthesis models, we extrapolate the observed fraction of Compton-thick AGNs to the intrinsic fraction. We find that the Compton-thick AGNs fraction is $20 \pm 3 \%$ of the type-II AGNs population.

Acknowledgements. We would like to thank the anonymous referee for comments and suggestions that helped to improve the paper. We acknowledge the use of MYTORUS spectral models by Yaqoob \& Murphy (2011). We would also like to thank Tahir Yaqoob for his help and suggestions in the application of the above models. The research leading to these results has received funding from the European Union's Horizon 2020 Programme under the AHEAD project (grant agreement n. 654215).

\section{References}

Akylas, A., Georgakakis, A., Georgantopoulos, I., Brightman, M., \& Nandra, K. 2012, A\&A, 546, A98

Akylas, A., Georgantopoulos, I., Ranalli, P., et al. 2016, A\&A, 594, A73

Annuar, A., Gandhi, P., Alexander, D. M., et al. 2015, ApJ, 815, 36

Arévalo, P., Bauer, F. E., Puccetti, S., et al. 2014, ApJ, 791, 81

Arnaud, K. A. 1996, ASP Conf. Ser., 101, 17

Baloković, M., Comastri, A., Harrison, F. A., et al. 2014, ApJ, 794, 111

Bauer, F. E., Arévalo, P., Walton, D. J., et al. 2015, ApJ, 812, 116

Baumgartner, W. H., Tueller, J., Markwardt, C. B., et al. 2013, ApJ, 207, 19

Brightman, M., \& Nandra, K. 2011, MNRAS, 413, 1206

Brightman, M., Nandra, K., Salvato, M., et al. 2014, MNRAS, 443, 1999

Buchner, J., Georgakakis, A., Nandra, K., et al. 2015, ApJ, 802, 89

Burlon, D., Ajello, M., Greiner, J., et al. 2011, ApJ, 728, 58

Churazov, E., Sunyaev, R., Revnivtsev, M., et al. 2007, A\&A, 467, 529

Comastri, A., Setti, G., Zamorani, G., \& Hasinger, G. 1995, A\&A, 296, 1 Dadina, M. 2007, A\&A, 461, 1209

Draper, A. R., \& Ballantyne, D. R. 2009, ApJ, 707, 778

Esposito, V., \& Walter, R. 2016, A\&A, 590, A49

Ferrarese, L., \& Merritt, D. 2000, ApJ, 539, L9

Frontera, F., Orlandini, M., Landi, R., et al. 2007, ApJ, 666, 86

Gehrels, N., Chincarini, G., Giommi, P., et al. 2004, ApJ, 611, 1005

Georgantopoulos, I., Comastri, A., Vignali, C., et al. 2013, A\&A, 555, A43

George, I. M., \& Fabian, A. C. 1991, MNRAS, 249, 352

Gilli, R., Comastri, A., \& Hasinger, G. 2007, A\&A, 463, 79

Gruber, D. E., Matteson, J. L., Peterson, L. E., \& Jung, G. V. 1999, ApJ, 520, 124

Harrison, F. A., Craig, W. W., Christensen, F. E., et al. 2013, ApJ, 770, 103

Ho, L. C., Filippenko, A. V., \& Sargent, W. L. W. 1997a, ApJS, 112, 315

Ho, L. C., Filippenko, A. V., \& Sargent, W. L. W. 1997b, ApJ, 487, 568

Koss, M. J., Romero-Cañizales, C., Baronchelli, L., et al. 2015, ApJ, 807, 149

Longair, M. S. 2011, High Energy Astrophysics

Magdziarz, P., \& Zdziarski, A. A. 1995, MNRAS, 273, 837

Malizia, A., Stephen, J. B., Bassani, L., et al. 2009, MNRAS, 399, 944

Marchesi, S., Ajello, M., Marcotulli, L., et al. 2018, ApJ, 854, 49

Masini, A., Comastri, A., Baloković, M., et al. 2016, A\&A, 589, A59

Masini, A., Civano, F., Comastri, A., et al. 2018, ApJS, 235, 17

Moretti, A., Pagani, C., Cusumano, G., et al. 2009, A\&A, 493, 501

Murphy, K. D., \& Yaqoob, T. 2009, MNRAS, 397, 1549

Nandra, K., \& Pounds, K. A. 1994, MNRAS, 268, 405

Puccetti, S., Comastri, A., Fiore, F., et al. 2014, ApJ, 793, 26

Puccetti, S., Comastri, A., Bauer, F. E., et al. 2016, A\&A, 585, A157

Ricci, C., Ueda, Y., Koss, M. J., et al. 2015, ApJ, 815, L13

Ricci, C., Trakhtenbrot, B., Koss, M. J., et al. 2017, ApJS, 233, 17

Titarchuk, L. 1994, ApJ, 434, 570

Treister, E., Urry, C. M., \& Virani, S. 2009, ApJ, 696, 110

Ueda, Y., Akiyama, M., Hasinger, G., Miyaji, T., \& Watson, M. G. 2014, ApJ, 786, 104

Vasudevan, R. V., Mushotzky, R. F., \& Gandhi, P. 2013, ApJ, 770, L37

Wada, K., Papadopoulos, P. P., \& Spaans, M. 2009, ApJ, 702, 63

Yaqoob, T. 2012, MNRAS, 423, 3360

Yaqoob, T., \& Murphy, K. D. 2011, MNRAS, 412, 1765 